\section{1-S06.03 PRE-EXPOSURE PROPHYLAXIS FOR HIV PREVENTION}

doi:10.1136/sextrans-2011-050109.33

${ }^{1} \mathrm{C}$ Cremin, ${ }^{1} \mathrm{~T}$ Hallett, ${ }^{2} \mathrm{M}$ Dybul, ${ }^{3} \mathrm{P}$ Piot, ${ }^{1} \mathrm{G}$ Garnett. ${ }^{1}$ Imperial College London, London, UK; ${ }^{2}$ Georgetown University, USA; ${ }^{3}$ London School of Hygiene and Tropical Medicine, $U K$

Background Two randomised controlled trials have recently provided evidence for the efficacy of Pre-Exposure Prophylaxis (PrEP), whereby uninfected individuals use antiretrovirals-either orally or topically-for the prevention of sexual acquisition of HIV. However, the potential population-level impact of a PrEP intervention remains uncertain.

Methods A population-based deterministic mathematical model was developed to investigate the impact of a PrEP intervention in Cotonou, Benin (1.7\% adult prevalence in 2006) and Kisumu, Kenya (13.9\% adult prevalence in 2008). It was assumed that PrEP has high intrinsic efficacy ( $90 \%$ reduction in acquisition per PrEP protected sex act) but its effectiveness is reduced by imperfect adherence $(75 \%$ of sex acts are protected for good adherers (40\% of PrEP users) and $30 \%$ of sex acts are protected for poor adherers ( $60 \%$ of PrEP users)). The impact of prioritising a fixed amount of $\operatorname{PrEP}$ with respect to behavioural risk was investigated, whereby $10 \%$ of the population received $\mathrm{PrEP}$ in each scenario.

Results In the absence of prioritisation of PrEP, an estimated $2.12 \%$ and $5.44 \%$ of infections would be averted over 10 years in Cotonou and Kisumu, respectively. However, the population-level epidemiological impact of a PrEP intervention can be substantially increased by prioritising to key populations most at risk of infection (Abstract O1-S06.03 figure 1). Percentage of infections averted over 10 years with respect to different levels of prioritisation by behavioural risk, in Cotonou, Benin and Kisumu, Kenya. The efficiency of increased prioritisation by behavioural risk is greater in Cotonou, as the HIV epidemic is more focussed within identifiable at-risk groups. However, the overall epidemiological impact of the PrEP intervention is greater in Kisumu, which has higher HIV incidence than Cotonou. Assuming an estimated cost range for PrEP of \$160-\$240 per-person per-year, the estimated cost per infection averted in Kisumu, Kenya, ranges from $\$ 13,578$ to $\$ 20,368$, if $50 \%$ of high risk individuals receive PrEP and the remaining PrEP is distributed among low and medium risk individuals. These costs should be considered in light of future averted costs of ART provision.

\section{Pre-Exposure Prophylaxis for HIV prevention}

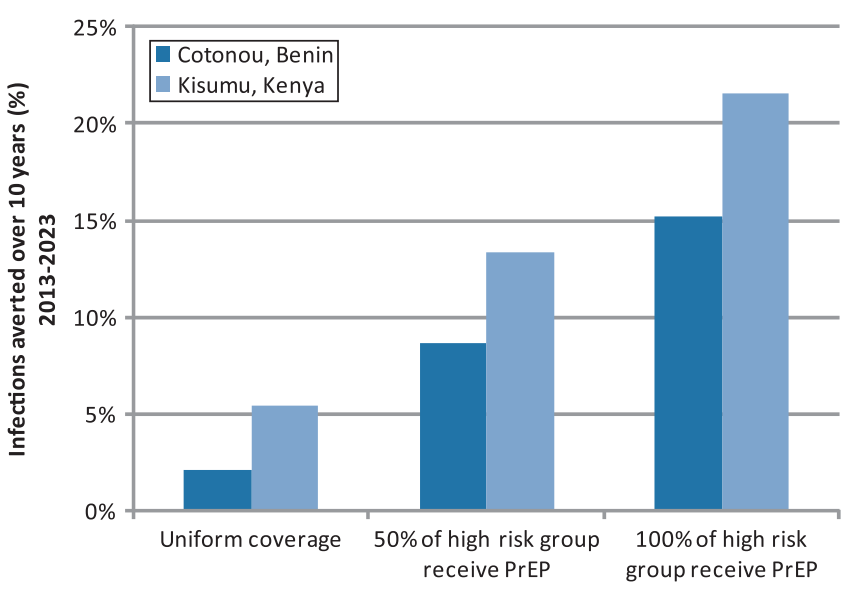

Abstract 01-S06.03 Figure 1 Percentage of infections averted over 10 years with respect to different levels of prioritisation by behavioural risk, in Cotonou, Benin and Kisumu, Kenya.

Conclusions An effective PrEP intervention could reduce the incidence of HIV transmission in generalised HIV epidemics. Consid- erable gains in impact, and therefore cost-effectiveness, can be achieved if PrEP is prioritised for those at highest risk of acquisition. Epidemiological context plays an important role in influencing the potential population-level impact of a PrEP intervention.

\section{1-S06.04 MODELLING THE EFFECTIVENESS OF COMBINATION PREVENTION FROM A HOUSE-TO-HOUSE HIV TESTING PLATFORM IN KWAZULU NATAL, SOUTH AFRICA}

doi:10.1136/sextrans-2011-050109.34

${ }^{1} \mathrm{R}$ Alsallaq, ${ }^{1} \mathrm{~J}$ Baeten, ${ }^{1} \mathrm{~J}$ Hughes, ${ }^{2} \mathrm{~L}$ Abu-Raddad, ${ }^{1} \mathrm{C}$ Celum, ${ }^{3} \mathrm{~T}$ Hallett. ${ }^{1}$ University of Washington, Seattle, USA; ${ }^{2}$ Weill Cornell Medical College, Oatar Doha, Oatar; ${ }^{3}$ Imperial College London, London, UK

Background Available HIV prevention interventions are partially efficacious, but might offer the best strategy to control HIV through synergistic combination and targeting. Home-based HIV testing and counselling (HBCT), conducted house-to-house, provides rapid population-level knowledge of HIV serostatus and a means to facilitate access to combined HIV prevention interventions: expedited referral to antiretroviral treatment (ART) for HIV+ persons, counselling for behaviour change, promotion of and referral for male circumcision and topical or oral PrEP for high-risk HIV-persons. Thus, massively conducted HBCT could serve as a platform for achieving high coverage of combination HIV prevention (HBCT-plus).

Methods We used age-sex stratified mathematical model to investigate the impact of HBCT-plus-including population-wide HBCT, behaviour change counselling, initiation of ART for HIV+ persons meeting national guidelines, topical PrEP, and male circumcision-on population-level HIV incidence. Our model was calibrated to data for KwaZulu-Natal province of South Africa. Effects of HBCT and other components of the HBCT-plus intervention package were based on empirical data from African settings.

Results Without major intervention efforts in this province, the model projects HIV incidence to be approximately $2 \%$ through the year 2020. In contrast, with high-coverage of HBCT-plus intervention the model indicates that incidence could be reduced by approximately half, to 1\%, under assumptions inAbstract O1-S06.04 table 1. Short and long term reductions in HIV incidence are mediated by short and long term behaviour changes following HBCT, respectively, and are manifested by repeated rounds of HBCT every 4 years. Longer-term impacts are supported by a reduction in infectiousness (due to timely initiation of ART as a result of earlier knowledge of serostatus because of HBCT) and susceptibility (due to circumcision) as well as associated 'herd immunity' effects.

Abstract 01-S06.04 Table 1 Uptake assumptions for combination HIV prevention (HBCT-plus)

\begin{tabular}{ll}
\hline HBCT intervention & \\
HBCT coverage & $90 \%$ \\
Linkage to ART intervention & \\
Uptake at $C D 4=350$ & $10 \%$ \\
Uptake at $C D 4=200$ & $70 \%$ \\
Uptake at $C D 4=50$ & $50 \%$ \\
Circumcision intervention & \\
Uptake over 4 years & $50 \%$ \\
\hline
\end{tabular}

Conclusions Multiple evidence-based HIV preventive interventions combined and delivered through a platform of periodic HBCT could reduce population-level HIV incidence in South Africa by $50 \%$ within 4 years. Fast and slow acting components affecting infectiousness and susceptibility can make combination packages particularly strong. Multiple partially-effective tools for HIV prevention can be combined and if coverage is sufficient, substantial reductions in HIV incidence are potentially within reach. 